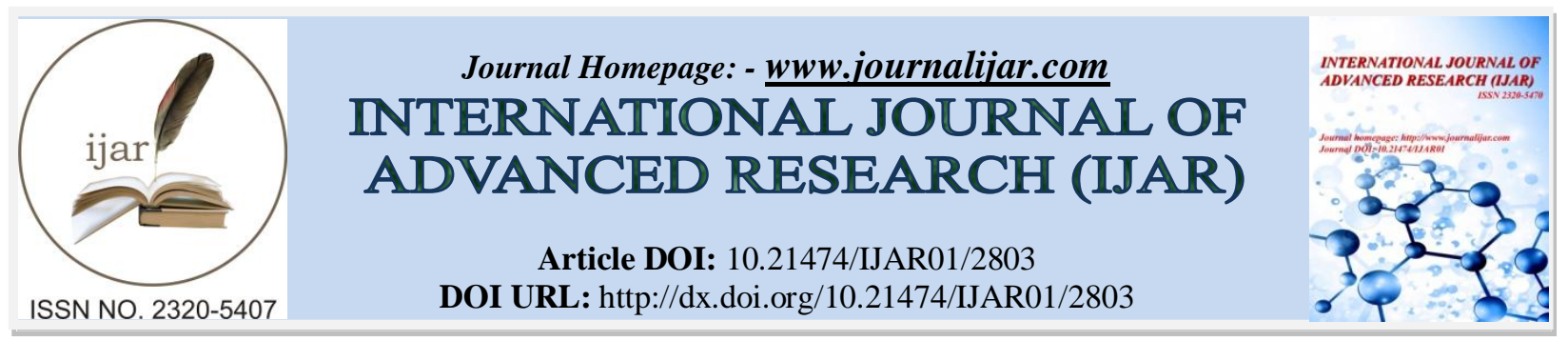

RESEARCH ARTICLE

\title{
SOME VERY SPECIAL PYTHAGOREAN TRIANGLES WITH THEIR PERIMETERS AS BOTH TRIANGULAR AND PENTAGONAL NUMBERS.
}

Dr. Mita Darbari

Head, Department of Mathematics St. Aloysius' College, Jabalpur, India

\section{Manuscript Info}

Manuscript History

Received: 15 November 2016

Final Accepted: 17 December 2016

Published: January 2017

Key words:-

Pythagorean Triangles, Triangular

Numbers, Pentagonal numbers.

\section{Abstract}

To find out the Special Pythagorean Triangles, where their perimeters are Triangular numbers and Pentagonal numbers both is the main objective of this paper. A few interesting results are observed.

\section{Introduction:-}

Figuret Numbers, first studied by Pythagoras, played very important role in Pythagorean science of numbers. Pythagorean Theorem, which gave the world, a powerful method of proof, reductio ad absurdum, also called Proof by Contradiction, continues to ignite the minds of those who love to play with numbers. Rana and Darbari [1] obtained special Pythagorean Triangles, with their legs to be consecutive, in terms of Triangular Numbers while Darbari [2] explored their perimeters as triangular numbers. Gopalan and Janaki [3] and Darbari [4] have studied special Pythagorean Triangles in terms of Pentagonal Numbers. Extending the problem further, existence of special Pythagorean triangles with their perimeters as Triangular Numbers and Pentagonal numbers both is explored in this paper.

\section{Method of Analysis:-}

The primitive solutions of the Pythagorean Equation,

$$
X^{2}+Y^{2}=Z^{2}
$$

is given by [5]

$$
X=m^{2}-n^{2}, Y=2 m n, Z=m^{2}+n^{2}
$$

for some integers $\boldsymbol{m}, \boldsymbol{n}$ of opposite parity such that $\boldsymbol{m}>\boldsymbol{n}>\mathbf{0}$ and $(m, n)=1$.

\section{Perimeter is a Triangular number:-}

Definition 2.1.1: A natural number $p$ is called a Triangular number if it can be written in the form

$$
\frac{\gamma(\gamma+1)}{2}, \gamma \in \mathrm{N}
$$

Definition 2.1.2: A natural number $p$ is called a Pentagonal number if it can be written in the form

$$
\frac{\beta(3 \beta-1)}{2}, \beta \in \mathrm{N}
$$

Corresponding Author:- Dr. Mita Darbari.

Address:- Head, Department of Mathematics St. Aloysius' College, Jabalpur, India. 
If the perimeter of the Pythagorean Triangle $(\mathrm{X}, \mathrm{Y}, \mathrm{Z})$ is Triangular and Pentagonal number $p$, then

$$
X+Y+Z=\frac{\gamma(\gamma+1)}{2}=\frac{\beta(3 \beta-1)}{2}=p
$$

By virtue of equation (2.2), equation (2.3) becomes

$$
2 m^{2}+2 m n=\frac{\gamma(\gamma+1)}{2}=\frac{\beta(3 \beta-1)}{2}=p, \gamma \& \beta \in N
$$

Using Mathematica for $0<\gamma<10^{20}, 0<\beta<10^{20}$, there are just 18 numbers which are both Triangular and Pentagonal numbers. They are as follows:

\begin{tabular}{|c|c|c|c|}
\hline S.N. & $\beta$ & $\gamma$ & $\frac{\gamma(\gamma+1)}{2}=\frac{\beta(3 \beta-1)}{2}$ \\
\hline 1. & 1 & 1 & 1 \\
\hline 2. & 12 & 20 & 210 \\
\hline 3. & 165 & 285 & 40755 \\
\hline 4. & 2296 & 3976 & 7906276 \\
\hline 5. & 31977 & 55385 & 1533776805 \\
\hline 6. & 445380 & 771420 & 297544793910 \\
\hline 7. & 6203341 & 10744501 & 57722156241751 \\
\hline 8. & 86401392 & 149651600 & 11197800766105800 \\
\hline 9. & 1203416145 & 2084377905 & 2172315626468283465 \\
\hline 10. & 16761424636 & 29031639076 & 421418033734080886426 \\
\hline 11. & 233456528757 & 404358569165 & 81752926228785223683195 \\
\hline 12. & 3251629977960 & 5631988329240 & 15859646270350599313653420 \\
\hline 13. & 45289363162681 & 78443478040201 & 3076689623521787481625080301 \\
\hline 14. & 630799454299572 & 1092576704233580 & 596861927316956420835951924990 \\
\hline 15. & 8785902997031325 & 15217630381229925 & 115788137209866023854693048367775 \\
\hline 16. & 122371842504138976 & 211954248632985376 & 22462301756786691671389615431423376 \\
\hline 17. & 1704419892060914337 & 2952141850480565345 & 4357570752679408318225730700647767185 \\
\hline 18. & 23739506646348661740 & 41118031658094929460 & 845346263718048427044120366310235410530 \\
\hline
\end{tabular}

Table 2.1:- Numbers which are both Triangular and Pentagonal

Since perimeter is even, investigating even such numbers from the Table 2.1 using software Mathematica, only the following six numbers have corresponding Pythagorean Triangles: 297544793910, 11197800766105800, $15859646270350599313653420,596861927316956420835951924990,22462301756786691671389615431423376$ and 845346263718048427044120366310235410530.

Case 1: For $\gamma=771420$ and $\beta=445380, \frac{\gamma(\gamma+1)}{2}=\frac{\beta(3 \beta-1)}{2}=297544793910,0<\mathrm{m}<10^{20}, 0<\mathrm{n}<10^{20}$, we get $07(\mathrm{X}, \mathrm{Y}, \mathrm{Z})$ which are given below:

Table 2.2:- (X, Y, Z) with $\mathrm{p}=297544793910$ (Triangular and Pentagonal No.)

\begin{tabular}{|l|l|l|l|l|l|l|}
\hline S.N. & $\mathrm{m}$ & $\mathrm{n}$ & $\mathrm{X}$ & $\mathrm{Y}$ & $\mathrm{Z}$ & $\mathrm{X}+\mathrm{Y}+\mathrm{Z}$ \\
\hline 1. & 275793 & 263642 & 35209270941 & 128804961460 & 133530561509 & 297544793910 \\
\hline 2. & 288535 & 227078 & 80300244885 & 93781558468 & 123462990557 & 297544793910 \\
\hline 3. & 290465 & 221722 & 86262074885 & 88031908068 & 123250810957 & 297544793910 \\
\hline 4. & 319189 & 146906 & 95049293885 & 78940172148 & 123555327877 & 297544793910 \\
\hline 5. & 323661 & 135994 & 134369999309 & 26262379860 & 136912414741 & 297544793910 \\
\hline 6. & 330609 & 119386 & 6554674685 & 145421236212 & 145568883013 & 297544793910 \\
\hline 7. & 368295 & 35654 & 31688028141 & 131039901460 & 134816864309 & 297544793910 \\
\hline
\end{tabular}


Table 2.3:- Verification of $\mathrm{X}^{2}+\mathrm{Y}^{2}=\mathrm{Z}^{2}$

\begin{tabular}{|l|l|l|l|}
\hline S.N. & $\mathrm{X}^{2}$ & $\mathrm{Y}^{2}$ & $\mathrm{X}^{2}+\mathrm{Y}^{2}=\mathrm{Z}^{2}$ \\
\hline 1. & 42963760226179849225 & 21147335941426300108944 & 21190299701652479958169 \\
\hline 2. & 1004131127464807915881 & 17171455774646510131600 & 18175586902111318047481 \\
\hline 3. & 1239692760196747025481 & 16590718096712085331600 & 17830410856908832357081 \\
\hline 4. & 6448129328590968663225 & 8794980708686902507024 & 15243110037277871170249 \\
\hline 5. & 7441145563465347763225 & 7749616838092803492624 & 15190762401558151255849 \\
\hline 6. & 9034368268037098393225 & 6231550778755874933904 & 15265919046792973327129 \\
\hline 7. & 18055296714300660477481 & 689712595910933619600 & 18745009310211594097081 \\
\hline
\end{tabular}

Case 2: For $\gamma=149651600$ and $\beta=86401392, \frac{\gamma(\gamma+1)}{2}=\frac{\beta(3 \beta-1)}{2}=11197800766105800,0<\mathrm{m}<10^{20}, 0<$ $\mathrm{n}<10^{20}$, we get $42(\mathrm{X}, \mathrm{Y}, \mathrm{Z})$ out of which first five are given below:

Table 2.4:- (X, Y, Z) with $\mathrm{p}=11197800766105800$ (Triangular and Pentagonal No.)

\begin{tabular}{|l|l|l|l|l|l|}
\hline S.N. & $\mathrm{m}$ & $\mathrm{n}$ & $\mathrm{X}$ & $\mathrm{Y}$ & $\mathrm{Z}$ \\
\hline 1. & 52932221 & 52842679 & 9471296095800 & 5594160726120118 & 5594168743889882 \\
\hline 2. & 53815300 & 50223893 & 373647086014551 & 5405627737925800 & 5418525942165449 \\
\hline 3. & 54351525 & 48661231 & 586172867390264 & 5289624226454550 & 5322003672260986 \\
\hline 4. & 54404196 & 48508829 & 606710051455175 & 5278167681292968 & 5312923033357657 \\
\hline 5. & 54418764 & 48466711 & 612379800130175 & 5274997015530408 & 5310423950445217 \\
\hline
\end{tabular}

Table 2.5:- Verification of $\mathrm{X}^{2}+\mathrm{Y}^{2}=\mathrm{Z}^{2}$ with $\mathrm{p}=11197800766105800$

\begin{tabular}{|c|c|c|c|}
\hline S.N. & $\mathrm{X}^{2}$ & $\mathrm{Y}^{2}$ & $\mathrm{X}^{2}+\mathrm{Y}^{2}=\mathrm{Z}^{2}$ \\
\hline 1. & 89705449734316322777640000 & $\begin{array}{l}31294634229664765872185764333 \\
924\end{array}$ & $\begin{array}{l}31294723935114500188508541973 \\
924\end{array}$ \\
\hline 2. & $\begin{array}{l}13961214488716527349698373 \\
1601\end{array}$ & $\begin{array}{l}29220811241032801487686305640 \\
000\end{array}$ & $\begin{array}{l}29360423385919966761183289371 \\
601\end{array}$ \\
\hline 3. & $\begin{array}{l}34359863046452402468608198 \\
9696\end{array}$ & $\begin{array}{l}27980124457094896460063215702 \\
500\end{array}$ & $\begin{array}{l}28323723087559420484749297692 \\
196\end{array}$ \\
\hline 4. & $\begin{array}{l}36809708653674109613503428 \\
0625\end{array}$ & $\begin{array}{l}27859054071845586219420246249 \\
024\end{array}$ & $\begin{array}{l}28227151158382327315555280529 \\
649\end{array}$ \\
\hline 5. & $\begin{array}{l}37500901960747308094694553 \\
0625\end{array}$ & $\begin{array}{l}27825593513854711458745572646 \\
464\end{array}$ & $\begin{array}{l}28200602533462184539692518177 \\
089\end{array}$ \\
\hline
\end{tabular}

Case 3: For $\gamma=5631988329240$ and $\beta=3251629977960, \frac{\gamma(\gamma+1)}{2}=\frac{\beta(3 \beta-1)}{2}=$

15859646270350599313653420, we get 23 (X, Y, Z) out of which first five are given below:

Table 2.6: (X, Y, Z) with p = 15859646270350599313653420 (Triangular and Pentagonal No.)

\begin{tabular}{|l|l|l|l|l|l|}
\hline S.N. & $\mathrm{m}$ & $\mathrm{n}$ & $\mathrm{X}$ & $\mathrm{Y}$ & $\mathrm{Z}$ \\
\hline 1. & 20218468 & 1900222303 & 47701986337804833599 & 76839169388940966709 & 76987094680784543067 \\
& 45270 & 403 & 2491 & 07620 & 53309 \\
\hline 2. & 20321607 & 1870002605 & 63276751956465102252 & 76002917454561647288 & 76265870053297835622 \\
& 37355 & 047 & 3816 & 61370 & 68234 \\
\hline 3. & 20648099 & 1775651417 & 11105023750846554183 & 73327656050470647122 & 74163782902188791830 \\
& 99165 & 809 & 36744 & 58970 & 57706 \\
\hline 4. & 20755084 & 1745156727 & 12621635156805721014 & 72441752316820759101 & 73533075229879513020 \\
& 96570 & 533 & 98811 & 23620 & 30989 \\
\hline 5. & 21011648 & 1672847921 & 16164736712979519174 & 70298585923800494170 & 72133140066725979792 \\
& 76678 & 267 & 30395 & 22052 & 00973 \\
\hline
\end{tabular}


Table 2.7:- $\mathrm{X}^{2}$ and $\mathrm{Y}^{2}$

\begin{tabular}{|l|l|l|}
\hline S.N. & $\mathrm{X}^{2}$ & $\mathrm{Y}^{2}$ \\
\hline 1. & 2275479500572119000279525960441573660900083 & 59042579523823624952392355159463208940074574 \\
& 85081 & 064400 \\
\hline 2. & 4003947338160010145652471147021989637542872 & 57764434616049115071458553580638467208256678 \\
& 01856 & 276900 \\
\hline 3. & 1233215525068660711246312063387769132271380 & 53769451418561245031525809224593719641000345 \\
& 521536 & 460900 \\
\hline 4. & 1593056740315141777140530505499837901792634 & 52478074787316058189023847851283404548003681 \\
& 413721 & 904400 \\
\hline 5. & 2612987129999479100589174330360538919819669 & 49418911828859609783418564170720369588487854 \\
& 856025 & 290704 \\
\hline
\end{tabular}

Table 2.8:- $X^{2}+Y^{2}=Z^{2}$ and $X+Y+Z=15859646270350599313653420$

\begin{tabular}{|l|l|l|}
\hline S.N. & $\mathrm{X}^{2}+\mathrm{Y}^{2}=\mathrm{Z}^{2}$ & $\mathrm{X}+\mathrm{Y}+\mathrm{Z}$ \\
\hline 1. & 59270127473880836852420307755507366306164582449481 & 15859646270350599313653420 \\
\hline 2. & 58164829349865116086023800695340666172010965478756 & 15859646270350599313653420 \\
\hline 3. & 55002666943629905742772121287981488773271725982436 & 15859646270350599313653420 \\
\hline 4. & 54071131527631199966164378356783242449796316318121 & 15859646270350599313653420 \\
\hline 5. & 52031898958859088884007738501080908508307524146729 & 15859646270350599313653420 \\
\hline
\end{tabular}

Case 4: For $\gamma=1092576704233580$ and $\beta=630799454299572, \frac{\gamma(\gamma+1)}{2}=\frac{\beta(3 \beta-1)}{2}=$ $596861927316956420835951924990,0<\mathrm{m}<10^{26}$ and $0<\mathrm{n}<10^{26}$, we get $50(\mathrm{X}, \mathrm{Y}, \mathrm{Z})$ out of which first ten are given below:

Table 2.9:- $(\mathrm{X}, \mathrm{Y}, \mathrm{Z})$ with $\mathrm{p}=$ (Triangular and Pentagonal No.)

\begin{tabular}{|l|l|l|l|l|l|}
\hline S.N. & $\mathrm{m}$ & $\mathrm{n}$ & $\mathrm{X}$ & $\mathrm{Y}$ & $\mathrm{Z}$ \\
\hline 1. & 38702757 & 3840569270 & 22906197744938832662 & 29728124139975896418 & 29729006614270357338 \\
& 3899585 & 09662 & 21817981 & 8625580540 & 1104526469 \\
\hline 2. & 38890000 & 3784719606 & 80021904883581102941 & 29437549633839799042 & 29448424049020032012 \\
& 7057443 & 53522 & 70693765 & 0948528492 & 0832702733 \\
\hline 3. & 39273313 & 3671491681 & 19440806726804549316 & 28838329051509049699 & 28903783007506137452 \\
& 8913605 & 51214 & 884422229 & 6643732940 & 2423769821 \\
\hline 4. & 39869520 & 3498238667 & 36581128009118528407 & 27894619584747699660 & 28133460346036089581 \\
& 4052845 & 18126 & 615642149 & 8846736940 & 9489545901 \\
\hline 5. & 39877996 & 3495800069 & 36819279347721045980 & 27881100604133135087 & 28123164192790402397 \\
& 5191097 & 94238 & 362862765 & 6495798172 & 9093264053 \\
\hline
\end{tabular}

Table 2.10:- $\mathrm{X}^{2}$ and $\mathrm{Y}^{2}$

\begin{tabular}{|l|l|l|}
\hline S.N. & $\mathrm{X}^{2}$ & $\mathrm{Y}^{2}$ \\
\hline 1. & $\begin{array}{l}52469389513024086275547257556686 \\
43721876815108694916361\end{array}$ & 88376136488181763109545570783716129636717530754052026691600 \\
\hline 2. & $\begin{array}{l}64035052611969011723463457972078 \\
835464765370181409875225\end{array}$ & 86656932844478168665653143651541636111498725799580135794064 \\
\hline 3. & $\begin{array}{l}37794496618896901461881806167163 \\
3414963576493407149328441\end{array}$ & 83164922248311084608572697422486591664011006888578041043600 \\
\hline 4. & $\begin{array}{l}13381789264195161097843955208300 \\
21602497785068541625338201\end{array}$ & 77810980177778992826348660996309110263321213562485560563600 \\
\hline 5. & $\begin{array}{l}13556593316855175331555433484441 \\
17070786121538786223445225\end{array}$ & 77735577089779307036137831051492572047797468613171358541584 \\
\hline
\end{tabular}


Table 2.11:- $X^{2}+Y^{2}=Z^{2}$ and $X+Y+Z=596861927316956420835951924990$

\begin{tabular}{|l|l|l|}
\hline $\mathrm{S} . \mathrm{N}$ & $\mathrm{X}^{2}+\mathrm{Y}^{2}=\mathrm{Z}^{2}$ & $\mathrm{X}+\mathrm{Y}+\mathrm{Z}$ \\
\hline 1. & 88381383427133065518173125509471798280439407569160721607961 & 596861927316956420835951924990 \\
\hline 2. & 86720967897090137677376607109513714946963491169761545669289 & 596861927316956420835951924990 \\
\hline 3. & 83542867214500053623191515484158225078974583381985190372041 & 596861927316956420835951924990 \\
\hline 4. & 79149159104198508936133056517139131865818998631027185901801 & 596861927316956420835951924990 \\
\hline 5. & 79091236421464824569293374399936689118583590151957581986809 & 596861927316956420835951924990 \\
\hline
\end{tabular}

Case 5: For $\gamma=211954248632985376$ and $\beta=122371842504138976, \frac{\gamma(\gamma+1)}{2}=\frac{\beta(3 \beta-1)}{2}=$

$5111330288523464647151142894071942298207292530339862362047627796736,0<\mathrm{m}<10^{30}$ and $0<\mathrm{n}<10^{30}$, we get $13(\mathrm{X}, \mathrm{Y}, \mathrm{Z})$ out of which first ten are given below:

Table 2.12:- (X, Y, Z) with $\mathrm{p}=$ (Triangular and Pentagonal No.)

\begin{tabular}{|l|l|l|l|l|l|}
\hline S.N. & $\mathrm{m}$ & $\mathrm{n}$ & $\mathrm{X}$ & $\mathrm{Y}$ & $\mathrm{Z}$ \\
\hline 1. & 78614658 & 6424865981 & 20523741689239888060 & $\begin{array}{l}10101772841608439904 \\
112816636603358\end{array}$ & $\begin{array}{l}10308154746254262961 \\
257807971535458\end{array}$ \\
& 03264125 & 9992643 & 18990823284560 & & \\
\hline 2. & 7 & & & 99534111879499203361 & 10214444523808142901 \\
& 95413662 & 6763707 & 43080040915792 & 72032364828094 & 174503025679490 \\
& 1 & & & & \\
\hline 3. & 81806709 & 5548216191 & 36140674711601137480 & 90776262331566167767 & 97706080524699611465 \\
& 76035546 & 4032547 & 72494097215312 & 57365286378334 & 59756047829730 \\
& 1 & & & & \\
\hline 4. & 87628440 & 4053943203 & 60352981039095566355 & 71048144488511581766 & 93221892040259768592 \\
& 89659342 & 9166633 & 01069026300240 & 29603097709518 & 58943307413618 \\
\end{tabular}

Table 2.13:- $\mathrm{X}^{2}$ and $\mathrm{Y}^{2}$

\begin{tabular}{|l|l|l|}
\hline S.N. & $\mathrm{X}^{2}$ & $\mathrm{Y}^{2}$ \\
\hline 1. & 421223972926643373884283467992496796675583 & 102045814543457854678865189716222350170701983 \\
& 5836639745386266734393600 & 648689758136091416876164 \\
\hline 2. & 526448265354751481953019630623111324910328 & 990703942764066443715293640382852192826055064 \\
& 5991584762312822034987264 & 03987514433554171672836 \\
\hline 3. & 130614836860976596181630161454925636718631 & 824032980288931874114656564139449109578590289 \\
& 99328749815107072887257344 & 51673303872370184615556 \\
\hline 4. & 364248232030542894834486219709878157010692 & 504783883526041865264271503078677090119292942 \\
& 55757302270604822624057600 & 98083756869857907792324 \\
\hline 5. & 378391094664185142552394373005524321141280 & 489376668854294719432810803043715542567582385 \\
& 22644963700347352765954304 & 81181784328555042529796 \\
\hline
\end{tabular}

Table 2.14:- $X^{2}+Y^{2}=Z^{2}$ and $X+Y+Z=22462301756786691671389615431423376$

\begin{tabular}{|c|c|c|}
\hline S.N. & $\mathrm{X}^{2}+\mathrm{Y}^{2}=\mathrm{Z}^{2}$ & $X+Y+Z$ \\
\hline 1. & $\begin{array}{l}1062580542727242884177080243961473181374578194853295035223 \\
58151269764\end{array}$ & $\begin{array}{l}2246230175678669167138961543142 \\
3376\end{array}$ \\
\hline 2. & $\begin{array}{l}1043348769299541591910595603445163325317087923955722767463 \\
76206660100\end{array}$ & $\begin{array}{l}2246230175678669167138961543142 \\
3376\end{array}$ \\
\hline 3. & $\begin{array}{l}9546478171499084702962867255943747462972222828042311897944 \\
3071872900\end{array}$ & $\begin{array}{l}2246230175678669167138961543142 \\
3376\end{array}$ \\
\hline 4. & $\begin{array}{l}8690321155565847600987577227885552471299855005538602747468 \\
0531849924\end{array}$ & $\begin{array}{l}2246230175678669167138961543142 \\
3376\end{array}$ \\
\hline 5. & $\begin{array}{l}8677677635184798619852051760492398637088626122614548467590 \\
7808484100\end{array}$ & $\begin{array}{l}2246230175678669167138961543142 \\
3376\end{array}$ \\
\hline
\end{tabular}


Case 6: For $\gamma=41118031658094929460$ and $\beta=23739506646348661740, \frac{\gamma(\gamma+1)}{2}=\frac{\beta(3 \beta-1)}{2}=$ $845346263718048427044120366310235410530,0<\mathrm{m}<10^{30}$ and $0<\mathrm{n}<10^{30}$, we get $1194(\mathrm{X}, \mathrm{Y}, \mathrm{Z})$ out of which first ten are given below: +

Table 2.15:- $(\mathrm{X}, \mathrm{Y}, \mathrm{Z})$ with $\mathrm{p}=$ (Triangular and Pentagonal No. $)$

\begin{tabular}{|l|l|l|l|l|l|}
\hline $\mathrm{S}$ & $\mathrm{m}$ & $\mathrm{n}$ & $\mathrm{X}$ & $\mathrm{Y}$ & $\mathrm{Z}$ \\
$\mathrm{N}$ & & & & & \\
\hline 1. & 1700707004 & 7845719448 & 22768511794395321582 & 26686540048804409642 & 35079574528605111479 \\
& 7924250415 & 912824176 & 2273080167337593249 & 8916610630980066080 & 2930675511917751201 \\
\hline 2. & 1776493775 & 6027610100 & 27926093005804577781 & 21416023654986583587 & 35192509711013681335 \\
& 9082954231 & 698743584 & 3182858666259636305 & 9863770185353807808 & 1073737458621966417 \\
\hline 3. & 1845860060 & 4439837949 & 32100777538029522056 & 16390639092947365976 & 36043209740827954672 \\
& 7691997615 & 068880496 & 0863735378436482209 & 0036435691904034080 & 3220195239894894241 \\
\hline 4. & 1514716915 & 1275726154 & 66689011279248760219 & 38647279702659762307 & 39218445541220204375 \\
& 2872275295 & 2606886672 & 228015989616101441 & 2695838569700736480 & 2196511750918572609 \\
\hline 5. & 1672132055 & 8556176557 & 20639440366740465628 & 28614114181307129275 & 35281071823757247800 \\
& 0497456663 & 615200992 & 3194551083005311505 & 6600141447109219392 & 4325673780120879633 \\
\hline
\end{tabular}

Table 2.16:- $X^{2}$ and $Y^{2}$

\begin{tabular}{|l|l|l|}
\hline S.N. & $\mathrm{X}^{2}$ & $\mathrm{Y}^{2}$ \\
\hline 1. & 518405129331518866650886685889121399347490 & 712171419776441662604698457844791506264620347 \\
& 86909392487499286743954367770376001 & 63235489599355021390321166566400 \\
\hline 2. & 779866670568847357927349350643338427219869 & 458646069190944906630902228093515495799154520 \\
& 37474752262854982822349270874053025 & 623359796379606663889925001764864 \\
\hline 3. & 103045991854606070335378694325477351851573 & 268653049875254452066329610731702601943794044 \\
& 050128048304847215752296722773519681 & 45650373767294601976177801446400 \\
\hline 4. & 444742422540376836197278922365527144233379 & 149361222841561724565620423677702648181947272 \\
& 1103610299361238167461283602276481 & 888786763299820469625854402790400 \\
\hline 5. & 425986498652235806314092518357488294012613 & 818767530380881765085221266137634200169038719 \\
& 41535439639411437100248042085365025 & 34115466996680922609323588849664 \\
\hline
\end{tabular}

Table 2.17:- $X^{2}+Y^{2}=Z^{2}$ and $X+Y+Z=845346263718048427044120366310235410530$

\begin{tabular}{|l|l|l|}
\hline S.N. & $\mathrm{X}^{2}+\mathrm{Y}^{2}=\mathrm{Z}^{2}$ & $\mathrm{X}+\mathrm{Y}+\mathrm{Z}$ \\
\hline 1. & 1230576549107960529255585143733912905612111216726279770986 & 8453462637180484270441203663102 \\
& 41765344688936942401 & 35410530 \\
\hline 2. & 12385127397597922645582515787368553923019023895370882424929 & 8453462637180484270441203663102 \\
& 43488738195875817889 & 35410530 \\
\hline 3. & 12991129684213151554201165539864776120459524545736986786145 & 8453462637180484270441203663102 \\
& 10354272900574966081 & 35410530 \\
\hline 4. & 1538086470669654929275932129013579196242810639923970626610 & 8453462637180484270441203663102 \\
& 58637087138005066881 & 35410530 \\
\hline 5. & 1244754029033117571399313784495122494181652134695551064081 & 8453462637180484270441203663102 \\
& 18022857365674214689 & 35410530 \\
\hline
\end{tabular}

\section{Observations and Conclusion:-}

We observe that

1. $\mathrm{X}+\mathrm{Y}+\mathrm{Z}=0(\bmod 2)$.

2. $Y+Z-X=0(\bmod 2)$.

3. $(\mathrm{X}+\mathrm{Y}+\mathrm{Z})(\mathrm{X}+\mathrm{Y}-\mathrm{Z})=0(\bmod 8)$.

4. $(\mathrm{Y}+\mathrm{Z}-\mathrm{X})^{2}=2(\mathrm{Y}+\mathrm{Z})(\mathrm{Z}-\mathrm{X})$.

5. $(X+2 Y+Z)^{2}=(Z-X)^{2}+4(X+Y)(Y+Z)$. 
In conclusion, other special Pythagorean Triangle can be found which satisfy the conditions other than discussed in the above problem.

\section{References:-}

1. Rana S.S., Darbari Mita; Special Pythagorean Triangles in terms of Triangular Numbers; Journal of Ultra Scientist of Physical Sciences; Vol. 23(2); 2011; 559-562

2. Darbari Mita; Special Pythagorean Triangles and Triangular Numbers; IJISET - International Journal of Innovative Science, Engineering \& Technology, Vol. 2 Issue 7, July 2015; 406-409.

3. Gopalan M.A., Janaki G.; Pythagorean triangles with perimeter as a pentagonal number; Antarctica J. Math.;5(2) (2008); 15-18.

4. Mita Darbari; Special Pythagorean Triangles and Pentagonal Numbers; Proceedings of $17^{\text {th }}$ Asian Technology Conference in Mathematics (ATCM 2012), December 16-20, 2012, organized by Suan Sunandha Raja Bhat University, Bangkok, Thailand;208-214

5. Ivan Niven, Herbert S. Zuckerman; An Introduction to the Theory of Numbers; Wiley Eastern Limited; New Delhi; 1976; Page No. 106. 